DOI: $10.3724 /$ SP.J.1218.2012.00704

\title{
双臂空间机器人捕获运动目标的自主路径规划
}

\author{
徐文福 1,2 , 刘厚德 1 , 李 成 3 , 张金涛 1,2 , 梁 斌 1,3 \\ （1. 哈尔滨工业大学深圳研究生院，广东 深圳 518055； 2. 深圳市数字化制造技术重点实验室, 广东 深圳 518055; \\ 3. 深圳航天东方红海特卫星有限公司, 广东 深圳 518057)
}

摘 要: 针对双臂空间机器人系统捕获运动目标的规划问题, 提出了自由漂浮和基座姿态受控两种模式下的自 主路径规划方法. 首先根据手眼相机测量结果, 规划各机械臂末端的运动速度, 然后采用阻尼最小二乘法求解所推 导的微分运动学方程, 得出各关节的期望角度和期望角速度, 作为关节控制器的输入, 直到满足抓捕条件. 最后建 立了双臂空间机器人系统的多体动力学模型并开展仿真研究, 仿真结果证明了所提方法的有效性.

关键词: 空间机器人; 双臂协调; 目标捕获; 路径规划

中图分类号: TP24 文献标识码: A 文章编号: 1002-0446(2012)-06-0704-11

\section{Autonomous Path Planning of Dual-arm Space Robot for Capturing Moving Target}

\author{
XU Wenfu ${ }^{1,2}$, LIU Houde ${ }^{1}$, LI Cheng ${ }^{3}$, ZHANG Jintao ${ }^{1,2}$, LIANG Bin ${ }^{1,3}$ \\ (1. Shenzhen Graduate School of Harbin Institute of Technology, Shenzhen 518055, China; \\ 2. Shenzhen Key Lab of Digital Manufacturing Technology, Shenzhen 518055, China; \\ 3. Aerospace Dongfanghong Development Ltd, Shenzhen 518057, China)
}

\begin{abstract}
For the case of dual-arm space robotic system capturing moving target, an autonomous path planning method is proposed corresponding to the two modes, i.e. the free-floating mode and the base attitude controlled mode. Firstly, the end-effector velocities of each arm are planned according to the hand-eye cameras' measurement. Then, the damped least squares (DLS) algorithm is used to solve the differential kinematics equations derived in this paper, and the desired angle and rate of each joint are determined. These desired values are utilized as the inputs of joint controllers. The process above will not end until the capturing condition is satisfied. Finally, the multi-body dynamic model of a dual-arm space robotic system is developed and simulation study is carried out. The simulation results verify the proposed methods.
\end{abstract}

Keywords: space robot; dual-arm coordination; target capturing; path planning

\section{1 引言 (Introduction)}

空间机器人在未来的空间活动中将扮演越来越 重要的角色，其应用领域广泛，包括卫星维修、大 型空间结构建造、轨道垃圾清理等，因而越来越受 到各航天大国的重视 ${ }^{[1]}$. 与单臂空间机器人系统 相比，双臂或多臂空间机器人具有更高灵活性，能 执行更加复杂的任务. 目前已装配到国际空间站上 的 Dextre ${ }^{[2]}$ 、Robonaut $2^{[3]}$, 即为双臂空间机器人系 统; Maryland 大学在 NASA 的资助下，也研制了具 有多臂的 Ranger 系统 ${ }^{[4]}$. 另外, 美国正在开展的 FREND 项目 ${ }^{[5]}$, 拟研制一套能对大多数非合作航天 器进行捕获和维修的多臂空间机器人系统.

双臂空间机器人系统的动力学、路径规划、
控制等比单臂空间机器人系统要复杂得多, 成为 近年来国内外研究的热点之一. 动力学与控制方 面, Papadopoulous 探讨了多臂空间机器人追踪及 捕获目标的动力学与控制问题 ${ }^{[6]}$. Moosavian 和 $\mathrm{Pa}$ padopoulos 提出了质心矢量法和直接路径法两种运 动学建模方法 ${ }^{[7]}$, 以及多臂柔顺控制方法 ${ }^{[8]} . \mathrm{Hu}$ 和 Vukovich 研究了形成封闭运动学链的多臂空间机器

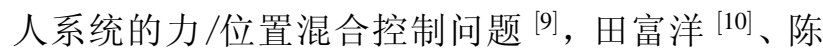
志煌 ${ }^{[11]}$ 、王从庆 ${ }^{[12]}$ 等也对闭链或柔性情况下双臂 空间机器人的动力学与控制开展了研究. 在路径 规划方面, Agrawal 提出了基于位置级逆运动学算 法的路径规划方法, 采用迭代搜索算法以满足角动 量守恒的条件 [13]. 陈力、郭益深将双向李亚普诺夫 
方法用于双臂空间机器人的非完整路径规划, 同 时控制基座姿态和机械臂关节角 ${ }^{[14-15]}, \mathrm{Wu}$ 等针对 柔性臂杆的情况，提出了基于 PSO (particle swarm optimization, 粒子群优化) 的振动抑制的轨迹规划 方法 ${ }^{[16]}$.

完整的目标抓捕过程（在此, “抓捕” 区别于 “捕 获”，包括从分离状态到最后控制手爪锁紧目标整 个过程）包括接触前的跟踪接近、接触碰撞、捕获 后的稳定 3 个阶段 ${ }^{[17]}$, 现有文献中也基本围绕这 三个方面的问题分别开展研究. 由于接触碰撞过程 及捕获后的稳定分析依赖于具体的手爪和被捕获机 构 ${ }^{[18-19]}$, 本文不作详细分析, 而主要集中在 “接触 前的跟踪接近” 直到末端工具对捕获进行包络这个 阶段的问题. Sagara 等人将广义雅可比矩阵用于双 臂空间机器人，提出自适应 RMRC (resolved motion rate control, 分解运动速度控制) 方法 ${ }^{[20]}$, 但所处理 的不是双臂同时捕获一个目标的情况，而是各自捕 获一个目标; Yamano 等建立了双臂捕获同一个目标 的地面实验系统，但基座为固定基座 ${ }^{[21]}$; 郭琦等 ${ }^{[22]}$ 开展了基座自由漂浮下的目标捕获研究, 但目标处 于静止状态. 另外, Takahashi 等建立了一套混合的 仿真系统, 对双臂捕获中的碰撞问题进行研究 ${ }^{[23]}$, Cong 等则分析了使得碰撞效应最小化的预捕获臂 型 ${ }^{[24]}$, 该方法也仅适用于目标特性（位置、姿态、运 动速度等) 确定的情况. 对于更加实际的情况, 目标 可能处于各种运动状态, 且其运动方向、速度大小 均会随时发生变化, 那些离线轨迹规划或基于目标 运动轨迹确知的方法将失效. 因此, 需要研究运动 目标自主捕获的路径规划方法. 本文对运动目标捕 获开展研究, 提出基座自由漂浮、基座姿态受控两 种典型模式下双臂空间机器人捕获运动目标的自主 路径规划方法, 并建立多体动力学仿真系统, 开展 仿真研究.

\section{2 问题描述 (Problem statement)}

假定待服务的航天器具有 3 个严重的机械故 障: (1) $+Z$ 向太阳翼未能成功展开; $(2)+Y$ 向通信 天线未能成功展开; (3) $-Y$ 向通信天线未能成功展 开. 此类故障比较复杂, 可采用如图 1 所示的双臂 空间机器人系统, 对其进行抓捕和维修. 该系统由 作为基座的航天器平台（称为空间基座或基座）、2 套机械臂 (称为空间机械臂) 以及对接锁紧机构等 组成. 2 套机械臂分别记为 $\operatorname{arm}-a$ 和 $a r m-b$, 协同完 成对故障卫星的在轨服务任务.

利用双臂开展在轨服务, 首先需要实现对运动
目标的自主捕获, 这即是本文所要探讨的问题, 该 问题可表述为:

问题（双臂协调捕获） 根据安装在机械臂末 端的手眼相机所提供的视觉测量结果, 自主规划、 控制双臂的运动, 使其同时捕获目标航天器.

双臂协调捕获的优点是双臂同时抓捕目标，有 2 个抓持点, 能实现目标航天器的快速稳定 (即目标 的运动速度快速衰减, 使得捕获后形成的复合体系 统能迅速稳定), 并使抓捕后的连接刚度更高. 根据 捕获过程中基座姿态是否受控，上述问题又可分为 如下 3 个子问题:

子问题 1 (基座自由漂浮模式下双臂协调捕获) 捕获过程中, 基座姿态及质心位置均不受控, 基座 处于自由漂浮状态. 在此模式下实现双臂的协调捕 获, 具有节约燃料、提高空间机器人服务寿命的优 点.

子问题 2 (基座姿态受控模式下双臂协调捕获) 捕获过程中, 基座姿态受控（在此仅考虑采用飞轮 作为执行机构的情况）、质心位置不受控. 在此模式 下实现双臂的协调捕获, 可为空间机器人提供比较 稳定的操作平台, 但由于飞轮控制力矩较小, 且容 易饱和，该模式的使用受到限制.

\section{子问题 3 （基座位姿受控模式下双臂协调捕获）} 捕获过程中, 基座姿态及质心位置均受控. 在此模 式下实现双臂的协调捕获, 使得空间机器人的规划、 控制方法与地面固定基座机器人类似, 但需要消耗 宝贵的燃料, 而且基于喷气系统的脉冲式控制, 很 难保证机械臂末端操作的稳定性, 所以一般不采用 此模式.
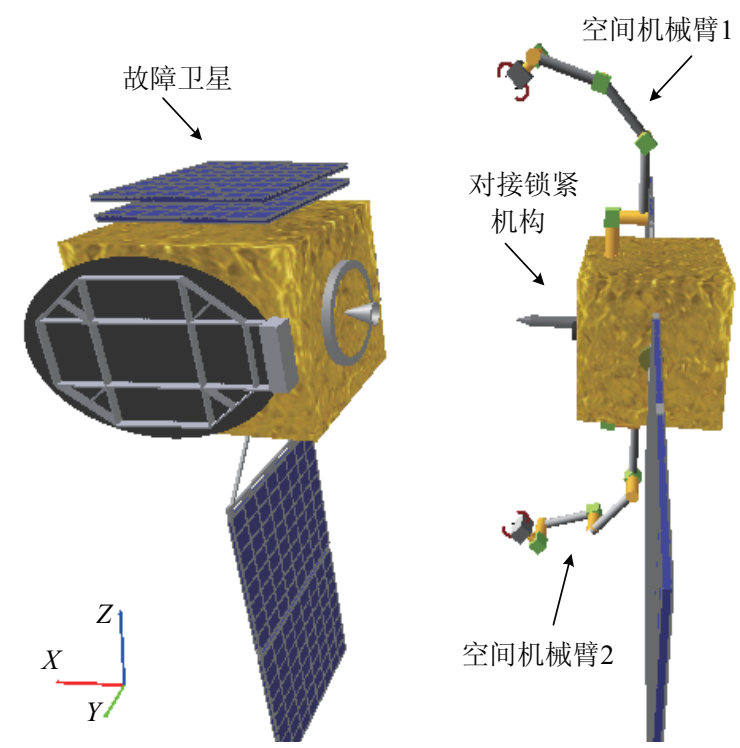

图 1 双臂空间机器人系统组成

Fig.1 The composition of a dual-arm space robotic system 


\section{3 双臂空间机器人系统建模（Modeling of a dual-arm space robotic system )}

不失一般性, 以平面双臂空间机器人系统为例, 建立其运动学方程. 完整描述刚体的平面运动需要 3 个自由度- 2 个平动自由度和 1 个转动自由度. 因此, 为实现末端的定位、定姿, 机械臂需要 3 个关 节. 图 2 为平面 3 关节双臂空间机器人系统的运动 学示意图. 为方便讨论, 定义如下符号: $B_{0}$ 表示基 座; $B_{i}^{k}(i=1, \cdots, 3)$ 为 $\operatorname{arm}-k(k=a, b$, 下同 $)$ 的第 $i$ 个连杆, $C_{i}^{k}$ 为 $B_{i}^{k}$ 的质心; $J_{i}^{k}$ 为 arm- $k$ 的第 $i$ 个关 节; $\boldsymbol{a}_{i}^{k}, \boldsymbol{b}_{i}^{k}$ 分别为从关节 $\boldsymbol{J}_{i}^{k}$ 指向 $C_{i}^{k} 、 C_{i}^{k}$ 指向 $J_{i+1}^{k}$ 的 位置矢量; $\boldsymbol{l}_{i}^{k}$ 为从 $\boldsymbol{J}_{i}^{k}$ 指向 $\boldsymbol{J}_{i+1}^{k}$ 的位置矢量, 且满足 $\boldsymbol{l}_{i}^{k}=\boldsymbol{a}_{i}^{k}+\boldsymbol{b}_{i}^{k} ; \varphi_{k}$ 为基座本体坐标系 $\Sigma_{0}$ 的 $X$ 轴到矢 量 $\boldsymbol{b}_{0}^{k}$ 的旋转角度, 由 $\mathrm{arm}-k$ 的安装位置确定. $\boldsymbol{r}_{0}$ 为 基座的质心位置矢量, $\boldsymbol{r}_{i}^{k}$ 为 $\operatorname{arm}-k$ 第 $i$ 个连杆的质 心位置矢量; $\theta_{0}$ 为基座姿态角, $\theta_{i}^{k}$ 为 $a r m-k$ 的 3 个 关节角. 另外, 图 2 中的相关坐标系定义如下:

$\Sigma_{\mathrm{I}}$ 即 $\left\{O_{\mathrm{I}} X_{\mathrm{I}} Y_{\mathrm{I}}\right\}$ : 惯性系, 其原点位于系统质心;

$\Sigma_{0}$ 即 $\left\{O_{0} X_{0} Y_{0}\right\}$ : 基座质心坐标系, 其原点位于 基座质心;

$\Sigma_{\mathrm{E} a} 、 \Sigma_{\mathrm{E} b}$ 即 $\left\{O_{\mathrm{e}}^{a} X_{\mathrm{e}}^{a} Y_{\mathrm{e}}^{a}\right\}$ 和 $\left\{O_{\mathrm{e}}^{b} X_{\mathrm{e}}^{b} Y_{\mathrm{e}}^{b}\right\}$ : 分别为 arm- $a$ 和 arm- $b$ 的末端坐标系;

$\Sigma_{\mathrm{T} a} 、 \Sigma_{\mathrm{T} b}$ 即 $\left\{O_{\mathrm{t}}^{a} X_{\mathrm{t}}^{a} Y_{\mathrm{t}}^{a}\right\}$ 和 $\left\{O_{\mathrm{t}}^{b} X_{\mathrm{t}}^{b} Y_{\mathrm{t}}^{b}\right\}$ : 为位于目 标航天器上的、分别由 arm- $a$ 和 arm- $b$ 的末端抓持 的目标手柄的坐标系.

\section{1 一般运动学方程}

定义如下变量 $(k=a, b$, 下同 $)$ :

$$
\theta_{0}^{k}=\theta_{0}+\varphi_{k}
$$

由图 2 可知, 各杆件质心的位置矢量为

$$
\boldsymbol{r}_{i}^{k}=\boldsymbol{r}_{0}+\boldsymbol{b}_{0}^{k}+\sum_{j=1}^{i-1} \boldsymbol{l}_{j}^{k}+\boldsymbol{a}_{i}^{k} \quad(i=1,2,3)
$$

各臂末端的位置矢量为

$$
\boldsymbol{p}_{\mathrm{e}}^{k}=\boldsymbol{r}_{0}+\boldsymbol{b}_{0}^{k}+\sum_{j=1}^{3} \boldsymbol{l}_{j}^{k}=\left[\begin{array}{c}
r_{0 x}+b_{0}^{k} c_{0}^{k}+\sum_{j=1}^{3} l_{j}^{k} c_{0 \ldots j}^{k} \\
r_{0 y}+b_{0}^{k} s_{0}^{k}+\sum_{j=1}^{3} l_{j}^{k} s_{0 \cdots j}^{k}
\end{array}\right]
$$

式中, 标量 $b_{0}^{k} 、 l_{i}^{k}$ 分别为相应矢量 $\boldsymbol{b}_{0}^{k} 、 \boldsymbol{l}_{i}^{k}$ 的长度, 且

$$
\begin{cases}s_{0}^{k}=\sin \theta_{0}^{k}, & c_{0}^{k}=\cos \theta_{0}^{k} \\ s_{0 \ldots j}^{k}=\sin \left(\sum_{i=0}^{j} \theta_{i}^{k}\right), & c_{0 \ldots j}^{k}=\cos \left(\sum_{i=0}^{j} \theta_{i}^{k}\right)\end{cases}
$$

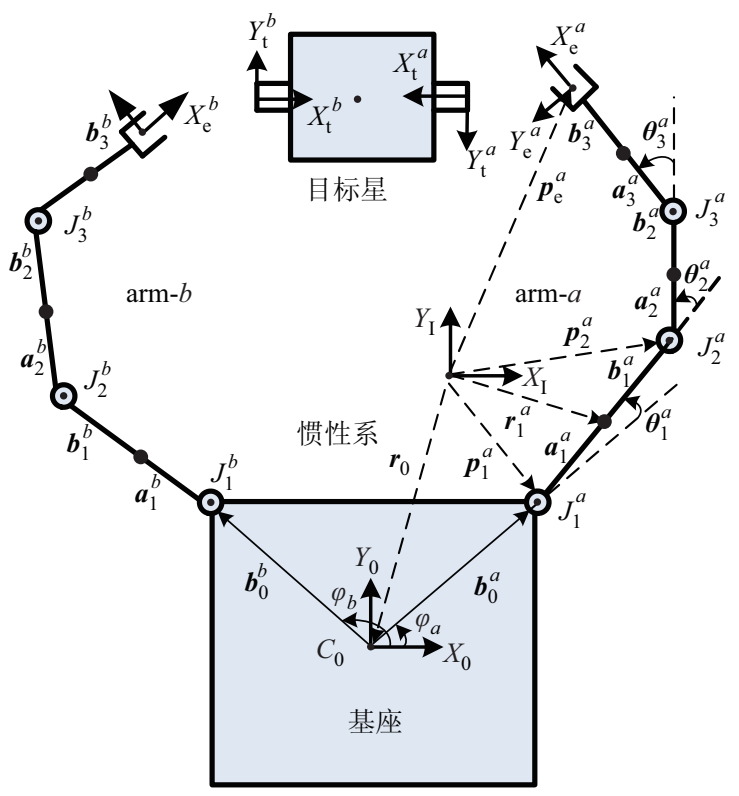

图 2 平面 3 关节双臂空间机器人系统一般模型

Fig.2 The general model of a planar dual-arm 3-joint space robotic system

对式 (2) 两边进行微分, 得到各杆件质心的线 速度为

$$
\boldsymbol{v}_{i}^{k}=\left[\begin{array}{c}
v_{0 x}-b_{0}^{k} s_{0}^{k} \dot{\theta}_{0}-\sum_{j=1}^{i-1} l_{j}^{k} s_{0 \ldots j}^{k} \dot{\theta}_{0 \cdots j}^{k}-a_{i}^{k} s_{0 \cdots i}^{k} \dot{\theta}_{0 \ldots i} \\
v_{0 y}+b_{0}^{k} c_{0}^{k} \dot{\theta}_{0}+\sum_{j=1}^{i-1} l_{j}^{k} c_{0 \ldots j}^{k} \dot{\theta}_{0 \ldots j}^{k}+a_{i}^{k} c_{0 \ldots i}^{k} \dot{\theta}_{0 \cdots i}
\end{array}\right]
$$

其中:

$$
\dot{\theta}_{0 \cdots j}^{k}=\sum_{i=0}^{j} \dot{\theta}_{i}^{k}
$$

对式 (3) 两边微分, 可得机械臂末端的线速度:

$$
\boldsymbol{v}_{\mathrm{e}}^{k}=\boldsymbol{J}_{\mathrm{s}_{-} \mathrm{v}}^{k} \dot{\boldsymbol{x}}_{b}+\boldsymbol{J}_{\mathrm{m}_{\mathrm{v}}}^{k} \dot{\Theta}^{k}
$$

其中:

$$
\begin{aligned}
\dot{\boldsymbol{x}}_{b}= & {\left[\begin{array}{lll}
v_{0 x} & v_{0 y} & \dot{\theta}_{0}
\end{array}\right]^{\mathrm{T}} } \\
\dot{\boldsymbol{\Theta}}^{k}= & {\left[\begin{array}{lll}
\dot{\theta}_{1}^{k} & \dot{\theta}_{2}^{k} & \dot{\theta}_{3}^{k}
\end{array}\right]^{\mathrm{T}} } \\
\boldsymbol{J}_{\mathrm{s}-\mathrm{v}}^{k}= & {\left[\begin{array}{ccc}
1 & 0 & -b_{0}^{k} s_{0}^{k}-\sum_{j=1}^{3} l_{j}^{k} s_{0 \ldots j}^{k} \\
0 & 1 & b_{0}^{k} c_{0}^{k}+\sum_{j=1}^{3} l_{j}^{k} c_{0 \ldots j}^{k}
\end{array}\right] } \\
\boldsymbol{J}_{\mathrm{m}_{-} \mathrm{v}}^{k}= & {\left[\begin{array}{ccc}
-\sum_{j=1}^{3} l_{j}^{k} s_{0 \cdots j}^{k} & -\sum_{j=2}^{3} l_{j}^{k} s_{0 \ldots j}^{k} & -l_{3}^{k} s_{0123}^{k} \\
\sum_{j=1}^{3} l_{j}^{k} c_{0 \cdots j}^{k} & \sum_{j=2}^{3} l_{j}^{k} c_{0 \ldots j}^{k} & l_{3}^{k} c_{0123}^{k}
\end{array}\right] }
\end{aligned}
$$


另一方面, 基座及各杆件的角速度分别为

$$
\begin{aligned}
& \omega_{0}=\dot{\theta}_{0} \\
& \omega_{i}^{k}=\dot{\theta}_{0}+\sum_{j=1}^{i} \dot{\theta}_{j}^{k} \quad(i=1, \cdots, 3)
\end{aligned}
$$

机械臂末端角速度为

$$
\omega_{\mathrm{e}}^{k}=\dot{\theta}_{0}+\sum_{j=1}^{n_{k}} \dot{\theta}_{j}^{k}
$$

$n_{k}$ 为第 $k$ 个机械臂关节个数. 联立式 (7)、式 (14), 有:

$$
\dot{\boldsymbol{x}}_{\mathrm{e}}^{k}=\left[\begin{array}{c}
\boldsymbol{v}_{\mathrm{e}}^{k} \\
\omega_{\mathrm{e}}^{k}
\end{array}\right]=\boldsymbol{J}_{\mathrm{s}}^{k} \dot{\boldsymbol{x}}_{b}+\boldsymbol{J}_{\mathrm{m}}^{k} \dot{\boldsymbol{\Theta}}^{k}
$$

其中:

$$
\begin{aligned}
& \boldsymbol{J}_{\mathrm{s}}^{k}=\left[\begin{array}{c}
\boldsymbol{J}_{\mathrm{s}_{-\mathrm{v}}}^{k} \\
{\left[\begin{array}{lll}
0 & 0 & 1
\end{array}\right]}
\end{array}\right] \\
& \boldsymbol{J}_{\mathrm{m}}^{k}=\left[\begin{array}{c}
\boldsymbol{J}_{\mathrm{m}_{-\mathrm{v}}}^{k} \\
{\left[\begin{array}{lll}
1 & 1 & 1
\end{array}\right]}
\end{array}\right]
\end{aligned}
$$

将两机械臂的运动学方程合一起可写成如下矩 阵形式:

$$
\left[\begin{array}{c}
\dot{\boldsymbol{x}}_{\mathrm{e}}^{a} \\
\dot{\boldsymbol{x}}_{\mathrm{e}}^{b}
\end{array}\right]=\left[\begin{array}{c}
\boldsymbol{J}_{\mathrm{s}}^{a} \\
\boldsymbol{J}_{\mathrm{s}}^{b}
\end{array}\right] \dot{\boldsymbol{x}}_{b}+\left[\begin{array}{c}
\boldsymbol{J}_{\mathrm{m}}^{a} \\
\boldsymbol{J}_{\mathrm{m}}^{b}
\end{array}\right]\left[\begin{array}{c}
\dot{\boldsymbol{\Theta}}^{a} \\
\dot{\boldsymbol{\Theta}}^{b}
\end{array}\right]
$$

式 (18) 即建立了双臂空间机器人系统的一般运动学 方程.

\section{2 基座姿态受控时的运动学方程}

空间机器人系统的线动量为

$$
\boldsymbol{P}=m_{0} \boldsymbol{v}_{0}+\sum_{i=1}^{3} m_{i}^{a} \boldsymbol{v}_{i}^{a}+\sum_{i=1}^{3} m_{i}^{b} \boldsymbol{v}_{i}^{b}
$$

将式 (5) 代入式 (19), 有:

$$
\boldsymbol{P}=\boldsymbol{H}_{b, \mathrm{p}} \dot{\boldsymbol{x}}_{b}+\boldsymbol{H}_{b \mathrm{~m}, \mathrm{p}}^{a} \dot{\boldsymbol{\Theta}}^{a}+\boldsymbol{H}_{b \mathrm{~m}, \mathrm{p}}^{b} \dot{\boldsymbol{\Theta}}^{b}
$$

其中:

$$
\begin{aligned}
\boldsymbol{H}_{b, \mathrm{p}} & =\left[\begin{array}{ccc}
M & 0 & k_{\mathrm{v} x} \\
0 & M & k_{\mathrm{v} y}
\end{array}\right] \\
\boldsymbol{H}_{b \mathrm{~m}, \mathrm{p}}^{k} & =\left[\begin{array}{lll}
k_{\mathrm{p} x 1}^{k} & k_{\mathrm{p} x 2}^{k} & k_{\mathrm{p} x 3}^{k} \\
k_{\mathrm{p} y 1}^{k} & k_{\mathrm{p} y 2}^{k} & k_{\mathrm{p} y 3}^{k}
\end{array}\right]
\end{aligned}
$$

式中各项的详细表达式见附录. 对于基座姿态受控 模式, 系统所受外力为 0 , 系统的线动量守恒, 假设 初始线动量为 0 , 则由式 (20) 可得:

$$
\boldsymbol{H}_{b, \mathrm{p}} \dot{\boldsymbol{x}}_{b}+\boldsymbol{H}_{b \mathrm{~m}, \mathrm{p}}^{a} \dot{\boldsymbol{\Theta}}^{a}+\boldsymbol{H}_{b \mathrm{~m}, \mathrm{p}}^{b} \dot{\boldsymbol{\Theta}}^{b}=0
$$

由于姿态受控, $\dot{\theta}_{0} \approx 0$, 代入式 (23), 可得:

$$
\boldsymbol{v}_{0}=-\frac{1}{M}\left(\boldsymbol{H}_{b \mathrm{~m}, \mathrm{p}}^{a} \dot{\boldsymbol{\Theta}}^{a}+\boldsymbol{H}_{b \mathrm{~m}, \mathrm{p}}^{b} \dot{\boldsymbol{\Theta}}^{b}\right)
$$

将 $\dot{\theta}_{0}=0$ 及式 (24) 代入式 (15), 可得:

$$
\begin{aligned}
& \dot{\boldsymbol{x}}_{\mathrm{e}}^{a}=\hat{\boldsymbol{J}}_{\mathrm{m}}^{a} \dot{\boldsymbol{\Theta}}^{a}+\check{\boldsymbol{J}}_{\mathrm{m}}^{b} \dot{\boldsymbol{\Theta}}^{b} \\
& \dot{\boldsymbol{x}}_{\mathrm{e}}^{b}=\breve{\boldsymbol{J}}_{\mathrm{m}}^{a} \dot{\boldsymbol{\Theta}}^{a}+\hat{\boldsymbol{J}}_{\mathrm{m}}^{b} \dot{\boldsymbol{\Theta}}^{b}
\end{aligned}
$$

其中:

$$
\begin{aligned}
& \hat{\boldsymbol{J}}_{\mathrm{m}}^{k}=\left[\begin{array}{c}
-\frac{1}{M} \boldsymbol{H}_{b \mathrm{~m}, \mathrm{p}}^{k}+\boldsymbol{J}_{\mathrm{m}, \mathrm{v}}^{k} \\
{\left[\begin{array}{lll}
1 & 1 & 1
\end{array}\right]}
\end{array}\right] \\
& \breve{\boldsymbol{J}}_{\mathrm{m}}^{k}=\left[\begin{array}{c}
-\frac{1}{M} \boldsymbol{H}_{b \mathrm{~m}, \mathrm{p}}^{k} \\
{\left[\begin{array}{lll}
0 & 0 & 0
\end{array}\right]}
\end{array}\right]
\end{aligned}
$$

式中 $\boldsymbol{J}_{\mathrm{m}, \mathrm{v}}^{k}$ 为 $\boldsymbol{J}_{\mathrm{m}}^{k}$ 的前两行元素组成的矩阵. 将式 (25) 和式 (26) 合并, 有:

$$
\left[\begin{array}{c}
\dot{\boldsymbol{x}}_{\mathrm{e}}^{a} \\
\dot{\boldsymbol{x}}_{\mathrm{e}}^{b}
\end{array}\right]=\tilde{\boldsymbol{J}}_{\mathrm{g}}\left[\begin{array}{c}
\dot{\boldsymbol{\Theta}}^{a} \\
\dot{\boldsymbol{\Theta}}^{b}
\end{array}\right]
$$

其中:

$$
\tilde{\boldsymbol{J}}_{\mathrm{g}}=\left[\begin{array}{ll}
\hat{\boldsymbol{J}}_{\mathrm{m}}^{a} & \breve{\boldsymbol{J}}_{\mathrm{m}}^{b} \\
\check{\boldsymbol{J}}_{\mathrm{m}}^{a} & \hat{\boldsymbol{J}}_{\mathrm{m}}^{b}
\end{array}\right]
$$

式 (29) 即为基座姿态受控模式下双臂空间机器人系 统的微分运动学方程.

\section{3 基座自由漂浮时的运动学方程} 系统的角动量为

$$
\begin{aligned}
\boldsymbol{L}= & \left(\boldsymbol{I}_{0} \omega_{0}+\boldsymbol{r}_{0} \times m_{0} \dot{r}_{0}\right)+\sum_{i=1}^{3}\left(\boldsymbol{I}_{i}^{a} \omega_{i}^{a}+\boldsymbol{r}_{i}^{a} \times m_{i}^{a} \dot{r}_{i}^{a}\right)+ \\
& \sum_{i=1}^{3}\left(\boldsymbol{I}_{i}^{b} \omega_{i}^{b}+\boldsymbol{r}_{i}^{b} \times m_{i}^{b} \dot{r}_{i}^{b}\right)
\end{aligned}
$$

\section{各杆件质心可表示为}

$$
\boldsymbol{r}_{i}^{k}=\boldsymbol{r}_{0}+\boldsymbol{r}_{0 i}^{k}
$$

式中, $\boldsymbol{r}_{0 i}^{k}$ 为基座质心到 $\mathrm{arm}-k$ 杆件 $i$ 质心的位置矢 量. 将式 (32) 代入式 (31), 有:

$$
\boldsymbol{L}=\boldsymbol{L}_{0}+\boldsymbol{r}_{0} \times \boldsymbol{P}
$$

其中,

$$
\begin{gathered}
\boldsymbol{L}_{0}=\boldsymbol{I}_{0} \boldsymbol{\omega}_{0}+\sum_{i=1}^{3}\left(\boldsymbol{I}_{i}^{a} \omega_{i}^{a}+\boldsymbol{r}_{0 i}^{a} \times m_{i}^{a} \dot{r}_{i}^{a}\right)+ \\
\sum_{i=1}^{3}\left(\boldsymbol{I}_{i}^{b} \boldsymbol{\omega}_{i}^{b}+\boldsymbol{r}_{0 i}^{b} \times m_{i}^{b} \dot{r}_{i}^{b}\right)
\end{gathered}
$$


当基座处于自由漂浮状态时, 整个系统的线动 量和角动量都守恒, 假设初始线动量和角动量均为 0 , 即

$$
\boldsymbol{L}=\boldsymbol{P}=0
$$

由式 (35) 和式 (33) 得:

$$
\boldsymbol{L}_{0}=0
$$

将式 (5)、(13) 代入式 (34), 经过化简, 有 (在平 面内只考虑 $Z$ 轴方向, 所以用标量表示):

$$
L_{0}=k_{\mathrm{v} x} v_{0 x}+k_{\mathrm{vy}} v_{0 y}+k_{\omega} \omega_{0}+\sum_{k=a, b} \sum_{i=1}^{3} k_{q i}^{a} \dot{\theta}_{i}^{k}=0
$$

式 (37) 中各项的详细表达式见附录. 联立线 动量守恒方程 (23) 和角动量守恒方程的等价形式 (37), 有:

$$
\boldsymbol{H}_{b} \boldsymbol{x}_{b}+\boldsymbol{H}_{b \mathrm{~m}}^{a} \dot{\boldsymbol{\Theta}}^{a}+\boldsymbol{H}_{b \mathrm{~m}}^{b} \dot{\boldsymbol{\Theta}}^{b}=0
$$

其中:

$$
\begin{aligned}
& \boldsymbol{H}_{b}= {\left[\begin{array}{ccc}
M & 0 & k_{\mathrm{v} x} \\
0 & M & k_{\mathrm{v} y} \\
k_{\mathrm{v} x} & k_{\mathrm{v} y} & k_{\omega}
\end{array}\right] } \\
& \boldsymbol{H}_{b \mathrm{~m}}^{k}=\left[\begin{array}{ccc}
k_{\mathrm{p} x 1}^{k} & k_{\mathrm{p} x 2}^{k} & k_{\mathrm{p} x 3}^{k} \\
k_{\mathrm{p} y 1}^{k} & k_{\mathrm{p} y 2}^{k} & k_{\mathrm{py} 3}^{k} \\
k_{q 1}^{k} & k_{q 2}^{k} & k_{q 3}^{k}
\end{array}\right]
\end{aligned}
$$

可以证明（由各项的表达式可知），矩阵 $\boldsymbol{H}_{b}$ 是可逆 的. 因此, 由式 (38) 可解出自由漂浮模式下基座运 动速度与各关节角速度的关系为

$$
\dot{\boldsymbol{x}}_{b}=\boldsymbol{J}_{b \mathrm{~m}}^{a} \dot{\boldsymbol{\Theta}}^{a}+\boldsymbol{J}_{b \mathrm{~m}}^{b} \dot{\boldsymbol{\Theta}}^{b}
$$

上式中：

$$
\boldsymbol{J}_{b \mathrm{~m}}^{k}=-\boldsymbol{H}_{b}^{-1} \boldsymbol{H}_{b \mathrm{~m}}^{k}
$$

将式 (41) 代入式 (15) 可分别得到两臂的运动速 度:

$$
\begin{aligned}
& \boldsymbol{v}_{\mathrm{e}}^{a}=\boldsymbol{J}_{a a}^{*} \dot{\boldsymbol{\Theta}}^{a}+\boldsymbol{J}_{a b}^{*} \dot{\boldsymbol{\Theta}}^{b} \\
& \boldsymbol{v}_{\mathrm{e}}^{b}=\boldsymbol{J}_{b a}^{*} \dot{\boldsymbol{\Theta}}^{a}+\boldsymbol{J}_{b b}^{*} \dot{\boldsymbol{\Theta}}^{b}
\end{aligned}
$$

其中:

$$
\begin{aligned}
\boldsymbol{J}_{a a}^{*} & =-\boldsymbol{J}_{\mathrm{s}}^{a} \boldsymbol{H}_{b}^{-1} \boldsymbol{H}_{b \mathrm{~m}}^{a}+\boldsymbol{J}_{\mathrm{m}}^{a} \\
\boldsymbol{J}_{a b}^{*} & =-\boldsymbol{J}_{\mathrm{s}}^{a} \boldsymbol{H}_{b}^{-1} \boldsymbol{H}_{b \mathrm{~m}}^{b} \\
\boldsymbol{J}_{b a}^{*} & =-\boldsymbol{J}_{\mathrm{s}}^{b} \boldsymbol{H}_{b}^{-1} \boldsymbol{H}_{b \mathrm{~m}}^{a} \\
\boldsymbol{J}_{b b}^{*} & =-\boldsymbol{J}_{\mathrm{s}}^{b} \boldsymbol{H}_{b}^{-1} \boldsymbol{H}_{b \mathrm{~m}}^{b}+\boldsymbol{J}_{\mathrm{m}}^{b}
\end{aligned}
$$

式 (43) 和式 (44) 可组合成下面的形式:

$$
\left[\begin{array}{c}
\dot{\boldsymbol{x}}_{\mathrm{e}}^{a} \\
\dot{\boldsymbol{x}}_{\mathrm{e}}^{b}
\end{array}\right]=\boldsymbol{J}_{\mathrm{g}}\left[\begin{array}{c}
\dot{\boldsymbol{\Theta}}^{a} \\
\dot{\boldsymbol{\Theta}}^{b}
\end{array}\right]
$$

式中:

$$
\boldsymbol{J}_{\mathrm{g}}=\left[\begin{array}{cc}
\boldsymbol{J}_{a a}^{*} & \boldsymbol{J}_{a b}^{*} \\
\boldsymbol{J}_{b a}^{*} & \boldsymbol{J}_{b b}^{*}
\end{array}\right]
$$

式 (49) 即建立了自由漂浮双臂空间机器人系统的微 分运动学方程, $\boldsymbol{J}_{\mathrm{g}}$ 为自由漂浮双臂空间机器人系统 的广义雅可比矩阵.

\section{4 双臂协调捕获的轨迹规划方法（Trajec- tory planning method for dual-arm coor- dinated capturing)}

\section{1 自主捕获的轨迹规划算法流程}

双臂协调捕获下，根据每个臂手眼相机所测出 的目标航天器抓捕手柄相对于其末端的位置, 自主 规划双臂的运动轨迹，使两臂以 “协调” 的方式同时 抓捕目标航天器. 捕获过程中, 手眼相机实时提供 目标相对于末端效应器的位置和姿态, 基于测量结 果，首先规划各机械臂末端的期望线速度和角速度， 然后根据运动学方程求解机械臂各关节角速度的期 望值。

所提出的双臂空间机器人捕获运动目标的自主 规划算法流程如图 3 所示.

主要步骤如下:

(1) 设置算法停止标志一一捕获区域阈值 $\varepsilon_{\mathrm{p}}$ 和 $\varepsilon_{0}$, 以及最大容许时长 $t_{\max } \cdot \varepsilon_{\mathrm{p}}$ 为相对位置误差 (单 轴)、 $\varepsilon_{\mathrm{o}}$ 为相对姿态误差, 在此分别取 $\varepsilon_{\mathrm{p}}=20 \mathrm{~mm}$ 和 $\varepsilon_{\mathrm{o}}=1^{\circ}$.

(2) 利用手眼相机测量目标相对于末端效应器 的位置 $\boldsymbol{p}_{\mathrm{et}}^{k}$ 和姿态 $\psi_{\mathrm{et}}^{k}(k=a, b)$.

(3) 根据手眼测量值, 计算相对位姿误差 (即 $\left.\boldsymbol{e}_{\mathrm{p}}^{k}, \boldsymbol{e}_{\mathrm{o}}^{k}\right)$, 并判断目标是否位于捕获区域内, 即 $\left\|\boldsymbol{e}_{\mathrm{p}}^{k}\right\| \leqslant$ $\varepsilon_{\mathrm{p}} 、\left\|\boldsymbol{e}_{\mathrm{o}}^{k}\right\| \leqslant \varepsilon_{\mathrm{o}}$. 若处于捕获区域内, 则闭合手爪、捕 获目标, 否则, 转第 (4) 步.

(4) 根据测量值预测目标的运动速度.

(5) 从相应的敏感器中读取空间机器人的当前 状态, 即 $\theta_{0}, \omega_{0}, \Theta^{a}, \dot{\Theta}^{a}, \Theta^{b}, \dot{\Theta}^{b}$.

(6) 根据视觉测量及估计结果, 规划机械臂末端 效应器的线速度和角速度, 以驱动末端效应器沿当 前状态下的最短路径 (直线) 接近、跟踪目标航天器 (考虑目标是运动的情况, 最短路径与目标当前状态 相关). 
（7）调用奇异回避处理算法, 确定各关节的期望 角速度 $\dot{\boldsymbol{\Theta}}_{\mathrm{d}}^{k}$, 并积分得到期望角度, 即 $\boldsymbol{\Theta}_{\mathrm{d}}^{k}$.

(8) 关节控制器以 $\boldsymbol{\Theta}_{\mathrm{d}}^{k}, \dot{\boldsymbol{\Theta}}_{\mathrm{d}}^{k}$ 作为输入, 产生关节 控制力矩, 作用于关节上, 控制关节按期望值运动.

(9) $t=t+\Delta t$. 若 $t \leqslant t_{\text {max }}$, 转第 (2) 步; 否则, 认 为在最大容许时间内空间机器人无法捕获目标, 算 法停止 (此种情况可能发生在目标航天器超出空间 机器人的工作空间的情况).

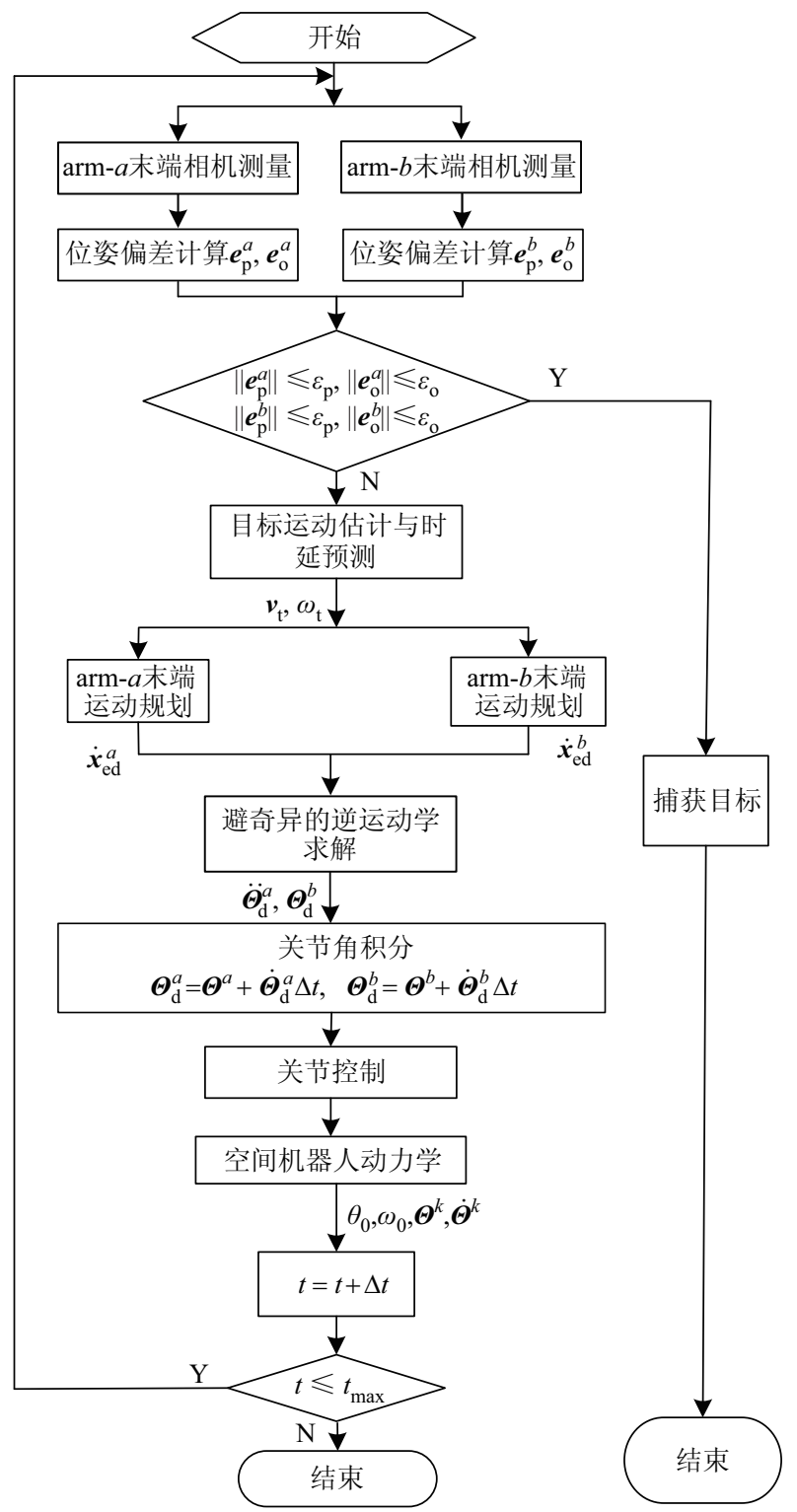

图 3 双臂空间机器人捕获运动目标的自主规划流程

Fig.3 The autonomous planning flowchart of the dual-arm space robot capturing a moving target

\section{2 相对位姿测量}

双臂空间机器人协调捕获任务中, 位于 arm- $a$ 和 $\operatorname{arm}-b$ 末端的相机分别提供目标相对于各自末端 的位置, 在仿真中表示为

$$
\boldsymbol{e}_{\mathrm{p}}^{k}=\boldsymbol{p}_{\mathrm{et}}^{k}=\boldsymbol{p}_{\mathrm{t}}^{k}-\boldsymbol{p}_{\mathrm{e}}^{k}
$$

其中, $\boldsymbol{p}_{\mathrm{t}}^{k}$ 为相应于 $a r m-k$ 手柄坐标系的原点在惯性 系下的位置, $\boldsymbol{p}_{\mathrm{e}}^{k}$ 为 $\mathrm{arm}-k$ 末端坐标系的原点在惯性 系下的位置.

手眼相机测量的相对姿态角为目标手柄坐标系 相对于机械臂末端坐标系的姿态角, 对于平面的情 况, 仅考虑绕 $Z$ 轴旋转的角度, 相对姿态角可用下 式表示:

$$
e_{\mathrm{o}}^{k}=\psi_{\mathrm{et}}^{k}=\psi_{\mathrm{t}}^{k}-\psi_{\mathrm{e}}^{k}
$$

式中, $\psi_{\mathrm{t}}^{k}$ 为 arm- $k$ 手柄坐标系相对于惯性系下 $Z$ 轴 的姿态角, $\psi_{\mathrm{e}}^{k}$ 为 $\mathrm{arm}-k$ 末端坐标系相对于惯性系下 $Z$ 轴的姿态角.

\section{3 空间机器人末端速度规划}

采用比例 - 微分的形式规划机械臂末端运动速 度, 即:

$$
\left\{\begin{array}{l}
\boldsymbol{v}_{\mathrm{ed}}^{k}=\boldsymbol{K}_{\mathrm{pv}}^{k}\left(\boldsymbol{p}_{\mathrm{t}}^{k}-\boldsymbol{p}_{\mathrm{e}}^{k}\right)+\boldsymbol{K}_{\mathrm{vv}}^{k} \boldsymbol{v}_{\mathrm{t}} \\
\omega_{\mathrm{ed}}^{k}=K_{\mathrm{p} \omega}^{k}\left(\theta_{\mathrm{t}}^{k}-\theta_{\mathrm{e}}^{k}\right)+K_{\mathrm{v} \omega}^{k} \omega_{\mathrm{t}}
\end{array}\right.
$$

式中, $\boldsymbol{K}_{\mathrm{pv}}^{k} 、 \boldsymbol{K}_{\mathrm{vv}}^{k} 、 K_{\mathrm{p} \omega}^{k} 、 K_{\mathrm{v} \omega}^{k}$ 为 arm- $k$ 视觉伺服控制的 比例、微分系数. 另一方面, 为避免末端速度过大, 采用下面的约束条件:

$$
\begin{aligned}
& \boldsymbol{v}_{\mathrm{ed}}^{k}= \begin{cases}\frac{v_{\mathrm{em}}}{\left|\boldsymbol{v}_{\mathrm{ed}}^{k}\right|} \boldsymbol{v}_{\mathrm{ed}}^{k}, & \text { if }\left|\boldsymbol{v}_{\mathrm{ed}}^{k}\right| \geqslant v_{\mathrm{em}} \\
\boldsymbol{v}_{\mathrm{ed}}^{k}, & \text { else }\end{cases} \\
& \omega_{\mathrm{ed}}^{k}= \begin{cases}\frac{\omega_{\mathrm{em}}}{\left|\omega_{\mathrm{ed}}^{k}\right|} \omega_{\mathrm{ed}}^{k}, & \text { if }\left|\omega_{\mathrm{ed}}^{k}\right| \geqslant \omega_{\mathrm{em}} \\
\omega_{\mathrm{ed}}^{k}, & \text { else }\end{cases}
\end{aligned}
$$

其中, $v_{\mathrm{em}} 、 \omega_{\mathrm{em}}$ 分别为允许的最大末端线速度和角 速度. 上述系数的取值主要用于限制末端运动速度 的大小, 系数取值越大则末端运动速度越大, 取值 越小则末端运动速度越小. 采用式 (54) 的限制条件 后, 可取 $\boldsymbol{K}_{\mathrm{pv}}^{k}=\boldsymbol{K}_{\mathrm{vv}}^{k}=\operatorname{diag}[1,1]$, 即为单位阵; $K_{\mathrm{p} \omega}^{k}=$ $K_{\mathrm{v} \omega}^{k}=1$.

\section{4 关节角和角速度规划}

\subsection{1 基座姿态受控下关节角轨迹规划}

基座姿态受控下, 双臂空间机器人系统的运动 学方程如式 (29) 所示. 因此, 当规划末端的期望运 动速度后, 可按下式规划机械臂关节角轨迹:

$$
\left[\begin{array}{c}
\dot{\boldsymbol{\Theta}}_{\mathrm{d}}^{a} \\
\dot{\boldsymbol{\Theta}}_{\mathrm{d}}^{b}
\end{array}\right]=\tilde{\boldsymbol{J}}_{\mathrm{g}}^{\#}\left[\begin{array}{c}
\dot{\boldsymbol{x}}_{\mathrm{ed}}^{a} \\
\dot{\boldsymbol{x}}_{\mathrm{ed}}^{b}
\end{array}\right]
$$

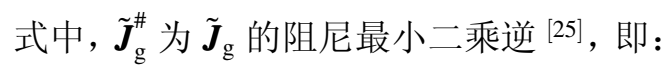

$$
\tilde{\boldsymbol{J}}_{\mathrm{g}}^{\#}=\left(\tilde{\boldsymbol{J}}_{\mathrm{g}}^{\mathrm{T}} \tilde{\boldsymbol{J}}_{\mathrm{g}}+\lambda^{2} \boldsymbol{I}\right)^{-1} \tilde{\boldsymbol{J}}_{\mathrm{g}}^{\mathrm{T}}
$$


按式 (66) 求解得到的是满足下式的最小二乘 解:

$$
\min _{\boldsymbol{\Theta}}\left(\left\|\dot{\boldsymbol{x}}_{\mathrm{ed}}-\tilde{\boldsymbol{J}}_{\mathrm{g}} \dot{\boldsymbol{\Theta}}_{\mathrm{d}}\right\|^{2}+\lambda^{2}\left\|\dot{\boldsymbol{\Theta}}_{\mathrm{d}}\right\|^{2}\right)
$$

采用 SVD 分解后可以看出其阻尼系数的含义

$$
\dot{\boldsymbol{\Theta}}_{\mathrm{d}}=\tilde{\boldsymbol{J}}_{\mathrm{g}}^{\#} \dot{\boldsymbol{x}}_{\mathrm{ed}}=\sum_{i=1}^{6} \frac{\sigma_{i}}{\sigma_{i}^{2}+\lambda^{2}} \boldsymbol{v}_{i} \boldsymbol{u}_{i}^{\mathrm{T}} \dot{\boldsymbol{x}}_{\mathrm{ed}}
$$

阻尼系数 $\lambda$ 一般按下式进行自适应调整:

$$
\lambda^{2}= \begin{cases}0, & \text { if } \hat{\sigma}_{6} \geqslant \varepsilon \\ \left(1-\left(\frac{\hat{\sigma}_{6}}{\varepsilon}\right)^{2}\right) \lambda_{\mathrm{m}}^{2}, & \text { otherwise }\end{cases}
$$

其中, $\hat{\sigma}_{6}$ 为最小奇异值的估计值, $\varepsilon$ 为选定的用于 判断机器人是否奇异的阈值, $\lambda_{\mathrm{m}}$ 为用户设定的奇异 区域的最大阻尼值. 在本文中, 取:

$$
\lambda_{\mathrm{m}}^{2}=0.05
$$

实际应用中, 需要对关节角速度进行限制, 即:

$$
\dot{\theta}_{i \mathrm{~d}}^{k}=\left\{\begin{array}{ll}
\frac{\dot{\theta}_{i \mathrm{~m}}^{k}}{\left|\dot{\theta}_{i \mathrm{~d}}^{k}\right|} \dot{\theta}_{i \mathrm{~d}}^{k}, & \text { if }\left|\dot{\theta}_{i \mathrm{~d}}^{k}\right| \geqslant \dot{\theta}_{i \mathrm{~m}}^{k} \\
\dot{\theta}_{i \mathrm{~d}}^{k}, & \text { else }
\end{array} \quad(i=1,2,3)\right.
$$

关节角期望值通过关节角速度期望值进行数值 积分得到，即：

$$
\left\{\begin{array}{l}
\boldsymbol{\Theta}_{\mathrm{d}}^{a}=\boldsymbol{\Theta}^{a}+\dot{\boldsymbol{\Theta}}_{\mathrm{d}}^{a} \Delta t \\
\boldsymbol{\Theta}_{\mathrm{d}}^{b}=\boldsymbol{\Theta}^{b}+\dot{\boldsymbol{\Theta}}_{\mathrm{d}}^{b} \Delta t
\end{array}\right.
$$

$\Delta t$ 为关节控制周期. 式 (56) 和式 (63) 即分别规划机 械臂关节角和角速度.

\subsection{2 自由漂浮模式下关节轨迹规划}

对于自由漂浮双臂空间机器人的情况, 运动学 方程如式 (49) 所示, 则期望的关节角速度可按下式 规划:

$$
\dot{\boldsymbol{\Theta}}_{\mathrm{d}}=\left[\begin{array}{c}
\dot{\boldsymbol{\Theta}}_{\mathrm{d}}^{a} \\
\dot{\boldsymbol{\Theta}}_{\mathrm{d}}^{b}
\end{array}\right]=\boldsymbol{J}_{\mathrm{g}}^{-1}\left[\begin{array}{c}
\dot{\boldsymbol{x}}_{\mathrm{ed}}^{a} \\
\dot{\boldsymbol{x}}_{\mathrm{ed}}^{b}
\end{array}\right]
$$

式中, $\dot{\boldsymbol{\Theta}}_{\mathrm{d}}^{a} 、 \dot{\boldsymbol{\Theta}}_{\mathrm{d}}^{b}$ 分别为 arm- $a$ 、 arm- $b$ 的期望角速度; $\boldsymbol{J}_{\mathrm{g}}$ 为双臂空间机器人系统的广义雅可比矩阵, 是当前 状态 $\left(\theta_{0}, \boldsymbol{\Theta}^{a}, \boldsymbol{\Theta}^{b}\right)$ 和各杆件质量特性 $\left(m_{i}, \boldsymbol{I}_{i}\right)$ 的函 数, 其奇异为动力学奇异. 当前状态可以通过相应 的传感器检测出. 式 (64) 有解的前提是广义雅可比 矩阵满秩, 对于广义雅可比矩阵奇异的情况, 可采 用 DLS 法进行奇异回避处理 ${ }^{[25]}$, 即:

$$
\dot{\Theta}_{\mathrm{d}}=\boldsymbol{J}_{\mathrm{g}}^{\#} \dot{\boldsymbol{x}}_{\mathrm{ed}}
$$

其中, $\boldsymbol{J}_{\mathrm{g}}^{\#}$ 为雅可比矩阵的最小二乘逆:

$$
\boldsymbol{J}_{\mathrm{g}}^{\#}=\left(\boldsymbol{J}_{\mathrm{g}}^{\mathrm{T}} \boldsymbol{J}_{\mathrm{g}}+\lambda^{2} \boldsymbol{I}\right)^{-1} \boldsymbol{J}_{\mathrm{g}}^{\mathrm{T}}
$$

按式 (66) 求解得到的是满足下式的最小二乘解

$$
\min _{\boldsymbol{\Theta}}\left(\left\|\dot{\boldsymbol{x}}_{\mathrm{ed}}-\boldsymbol{J}_{\mathrm{g}} \dot{\boldsymbol{\Theta}}_{\mathrm{d}}\right\|^{2}+\lambda^{2}\left\|\dot{\boldsymbol{\Theta}}_{\mathrm{d}}\right\|^{2}\right)
$$

阻尼系数 $\lambda$ 的选择与式 (60) 相同. 规划出关节的期 望角速度后, 其余处理过程同式 (62) 和 (63).

需要指出的是, 对于双臂空间机器人系统的奇 异回避, 也可采用文 [26] 提出的实用的动力学奇异 回避方法进行处理.

\section{5 仿真研究 (Sumulation study)}

\section{1 仿真模型的建立}

空间机器人由基座、2 个 3 连杆机械臂（arm- $a$ 、 arm- $b$ ）组成, 假设基座为 $1 \mathrm{~m} \times 1 \mathrm{~m} \times 1 \mathrm{~m}$ 的正方体, 目标航天器为 $0.5 \mathrm{~m} \times 0.5 \mathrm{~m} \times 0.5 \mathrm{~m}$ 的正方体, 手柄高 出两侧 $0.1 \mathrm{~m}$ 的距离, 质心位于几何中心. 整个双臂 空间机器人系统的质量特性参数如表 1 所示. 另外, 基座质心到 arm- $a$ 和 arm- $b$ 安装位置的连线与基座 质心坐标系 $X$ 轴的夹角分别为 $\varphi_{a}=45^{\circ} 、 \varphi_{b}=135^{\circ}$. 为对所提出的方法进行验证, 利用 Matlab/Simulink 建立平面双臂空间机器人的动力学仿真系统, 如图 4 所示. 图中, $\Phi_{\mathrm{t}}$ 为目标的姿态角, $\Phi_{\mathrm{e}}^{a} 、 \Phi_{\mathrm{e}}^{b}$ 分别为 $\operatorname{arm}-a 、 \operatorname{arm}-b$ 末端的姿态角.

表 1 平面双臂空间机器人系统的质量特性参数

Tab.1 The mass property parameters of the planar dual-arm space robotic system

\begin{tabular}{c|ccccccc}
\hline & $S a t$ & $B_{1 \_a}$ & $B_{2 \_a}$ & $B_{3 \_a}$ & $B_{1 \_b}$ & $B_{2 \_b}$ & $B_{3 \_b}$ \\
\hline mass $/ \mathrm{kg}$ & 480 & 20 & 20 & 15 & 20 & 20 & 15 \\
\hline \multirow{2}{*}{${ }^{i} \boldsymbol{a}_{i} / \mathrm{m}$} & - & 0.375 & 0.375 & 0.25 & 0.375 & 0.375 & 0.25 \\
\cline { 2 - 8 } & - & 0 & 0 & 0 & 0 & 0 & 0 \\
\hline \multirow{2}{*}{${ }^{i} \boldsymbol{b}_{i} / \mathrm{m}$} & 0.5 & -0.5 & 0.375 & 0.375 & 0.25 & 0.375 & 0.375 \\
\cline { 2 - 8 } & 0.5 & 0.5 & 0 & 0 & 0 & 0 & 0 \\
\hline $\boldsymbol{I}_{i}$ & 100 & 2.4 & 2.4 & 2.0 & 2.4 & 2.4 & 2.0 \\
\hline
\end{tabular}

\section{2 仿真初始条件}

利用上面所建立的双臂空间机器人仿真系统， 对双臂协调捕获的自主路径规划方法进行仿真验 证. 初始时刻基座质心位置、基座姿态角、 $\operatorname{arm}-a$ 关 节角、 $a r m-b$ 关节角分别为

$$
\begin{aligned}
& \boldsymbol{r}_{00}=\left[\begin{array}{ll}
0, & 0.5
\end{array}\right]^{\mathrm{T}} \\
& \theta_{00}=0 \\
& \boldsymbol{\Theta}_{0}^{a}=\left[-15^{\circ}, 55^{\circ}, 80^{\circ}\right]^{\mathrm{T}} \\
& \boldsymbol{\Theta}_{0}^{b}=\left[15^{\circ},-55^{\circ},-80^{\circ}\right]^{\mathrm{T}}
\end{aligned}
$$




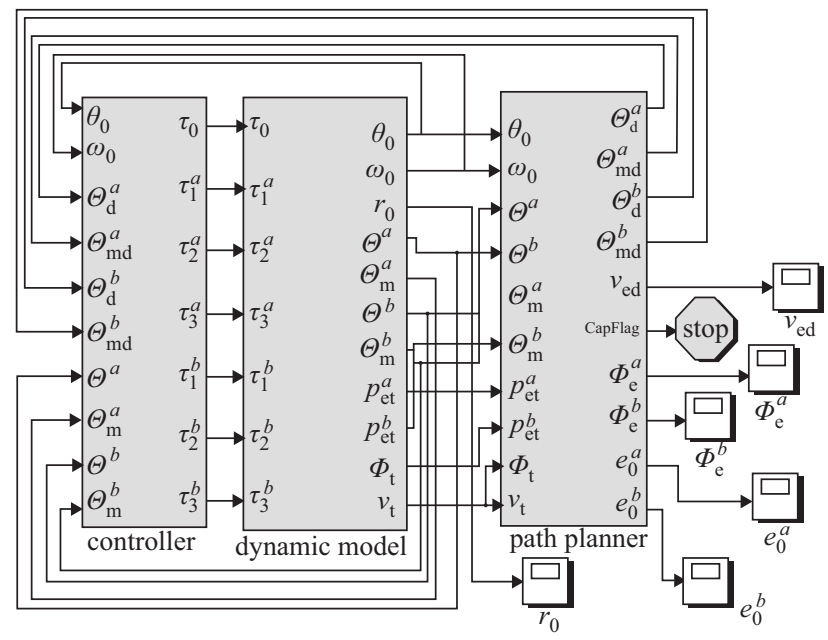

图 4 平面 3 连杆双臂空间机器人仿真系统组成

Fig.4 The composition of the simulation system of a planar dual-arm-3-link space robotic system

目标航天器的质心位置和姿态角分别为

$$
\begin{aligned}
& \boldsymbol{r}_{\mathrm{t} 0}=\left[\begin{array}{ll}
0.05, & 2.15
\end{array}\right]^{\mathrm{T}} \\
& \theta_{00}=3^{\circ}
\end{aligned}
$$

整个任务中, 目标航天器以下列速度运动:

$$
\begin{aligned}
& \boldsymbol{v}_{\mathrm{t} 0}=\left[\begin{array}{ll}
-10 \mathrm{~mm} / \mathrm{s} & 10 \mathrm{~mm} / \mathrm{s}
\end{array}\right]^{\mathrm{T}} \\
& \omega_{\mathrm{t} 0}=0.5^{\circ} / \mathrm{s}
\end{aligned}
$$

其中 $\boldsymbol{v}_{\mathrm{t} 0} 、 \omega_{\mathrm{t} 0}$ 分别为航天器质心坐标系的线速度和 角速度. 相应于上述初始条件的状态如图 5 所示 (SimMechanics 工具箱的仿真动画界面显示).

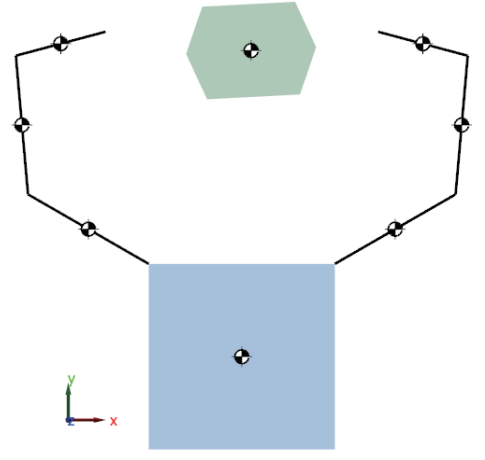

图 5 双臂协调捕获的初始状态

Fig.5 The initial state of the dual-arm coordinated capturing

\section{3 自由漂浮模式下双臂协调捕获仿真}

双臂协调捕获的仿真结果如图 $6 \sim 9$ 所示. 其 中, 图 6 和 7 分别为捕获过程中目标上捕获点 $a$ 和 捕获点 $b$ 相对于 $\operatorname{arm}-a$ 和 arm- $b$ 末端的位姿变化曲 线, 相对位姿不断趋近于 0 , 意味着机械臂末端逐渐 接近目标, 当 $t_{\mathrm{f}}=8.94 \mathrm{~s}$ 时,

$$
\begin{aligned}
& \boldsymbol{p}_{\mathrm{et}}^{a}\left(t_{\mathrm{f}}\right)=\left[\begin{array}{ll}
-0.0184, & 0.0073
\end{array}\right]^{\mathrm{T}} \\
& \boldsymbol{p}_{\mathrm{et}}^{b}\left(t_{\mathrm{f}}\right)=\left[\begin{array}{ll}
-0.0007, & -0.0009
\end{array}\right]^{\mathrm{T}}
\end{aligned}
$$

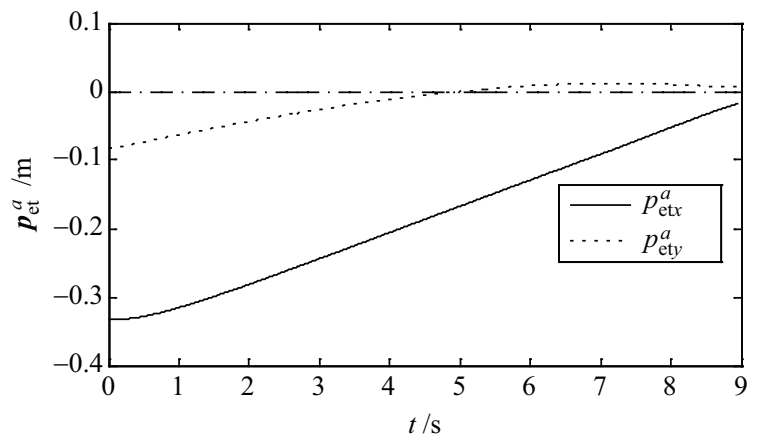

(a) The relative postion

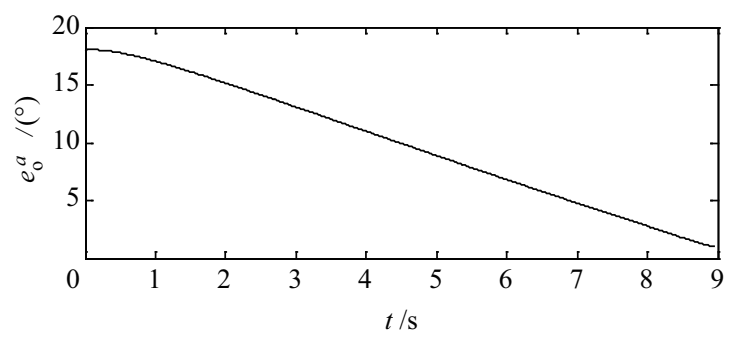

(b) The relative attitude

图 6 捕获过程中捕获点 $a$ 相对于 arm- $a$ 末端的位姿

Fig.6 The pose of capturing point $a$ with respect to arm- $a$ 's end-effector during capturing

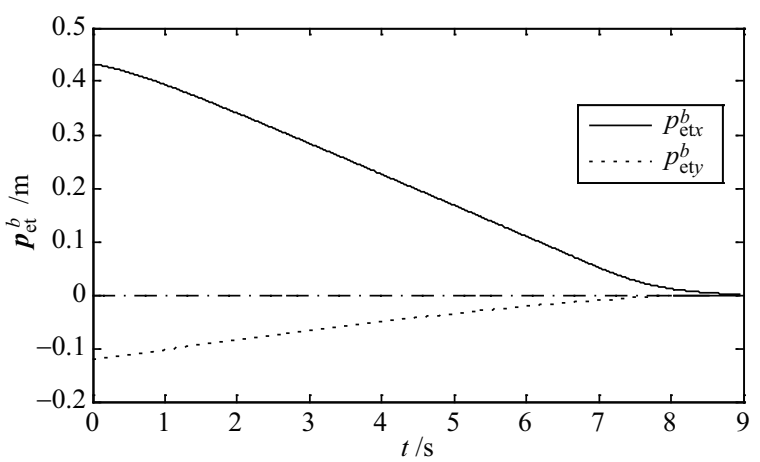

(a) The relative position

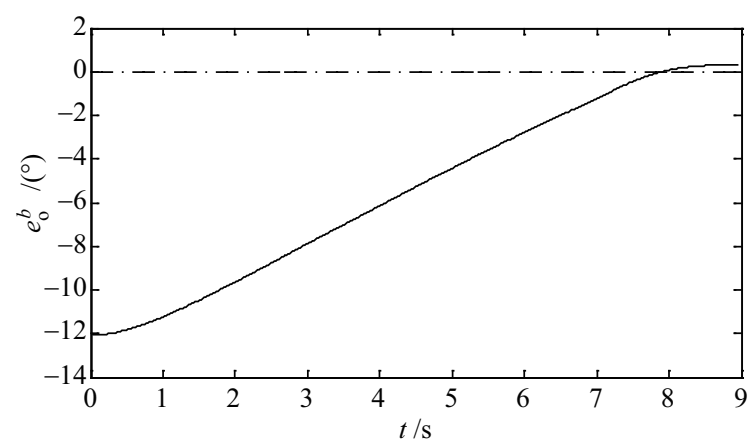

(b) The relative attitude

图 7 捕获过程中捕获点 $b$ 相对于 $\operatorname{arm}-b$ 末端的位姿

Fig.7 The pose of capturing point $b$ with respect to arm- $b$ 's end-effector during capturing

目标上的捕获点均在 $\operatorname{arm}-a$ 和 $\operatorname{arm}-b$ 末端的捕 获范围内 $\left(\varepsilon_{\mathrm{p}}=20 \mathrm{~mm}, \varepsilon_{\mathrm{o}}=1^{\circ}\right)$, 满足抓捕条件, 机 械臂可闭合手爪抓捕目标，仿真结束.

捕获过程中, arm- $a$ 关节角的变化曲线分别如图 8 所示. 整个抓捕过程中, 基座的姿态及质心位置均 
不受控, 而是在耦合动力学作用下运动, 其中, 基座 姿态从 $0^{\circ}$ 偏转到了 $-7.63^{\circ}$; 质心位置从 $[0,0.5] \mathrm{m}^{\mathrm{T}}$ 运动到了 $[0.0077 \mathrm{~m}, 0.4830 \mathrm{~m}]^{\mathrm{T}}$, 变化了 $[0.0077 \mathrm{~m}$, $-0.0170 \mathrm{~m}]^{\mathrm{T}}$. 捕获时刻如图 9 所示.
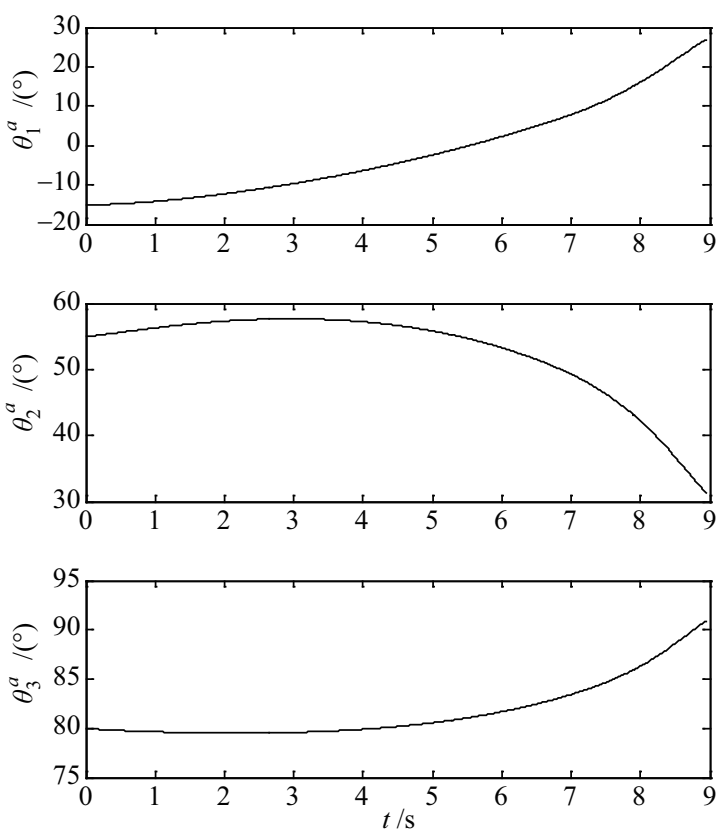

图 8 捕获过程中 arm- $a$ 关节运动轨迹

Fig. 8 The trajectory pose of arm- $a$ 's joints during capturing

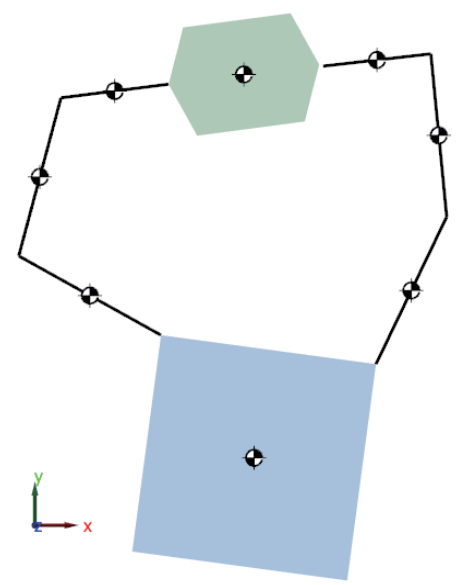

图 9 双臂协调捕获的最终状态

Fig.9 The final state of the dual-arm coordinated capturing

\section{4 基座姿态受控模式下双臂协调捕获仿真}

初始条件与上节相同, 但基座姿态采用飞轮进 行控制. 双臂协调捕获的仿真结果如图 $10 \sim 12$ 所 示. 其中, 图 10、11 分别为捕获过程中目标上捕获 点 $a$ 和捕获点 $b$ 相对于 arm- $a$ 和 arm- $b$ 末端的位姿 变化曲线, 相对位姿不断趋近于 0 , 意味着机械臂末 端逐渐接近目标, 当 $t_{\mathrm{f}}=9.09 \mathrm{~s}$ 时,

$$
\begin{aligned}
& \boldsymbol{p}_{\mathrm{et}}^{a}\left(t_{\mathrm{f}}\right)=\left[\begin{array}{ll}
-0.0188, & 0.0078
\end{array}\right]^{\mathrm{T}} \\
& \boldsymbol{p}_{\mathrm{et}}^{b}\left(t_{\mathrm{f}}\right)=\left[\begin{array}{ll}
-0.0048, & -0.0003
\end{array}\right]^{\mathrm{T}}
\end{aligned}
$$

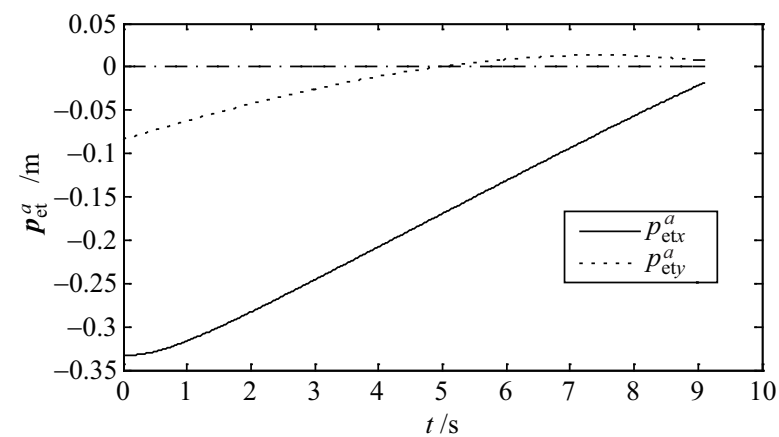

(a) The relative position

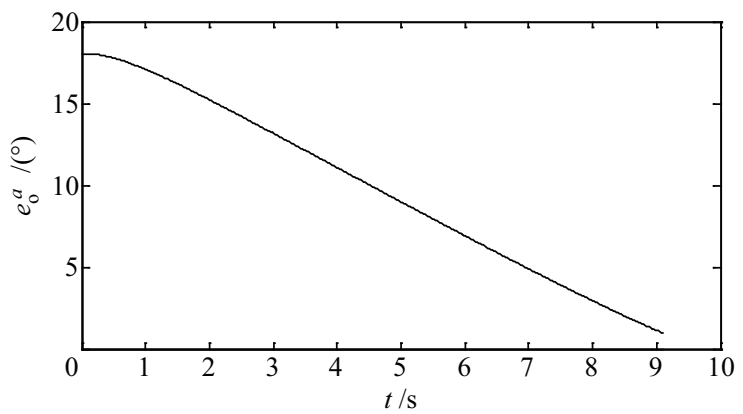

(b) The relative attitude

图 10 捕获过程中捕获点 $a$ 相对于 arm- $a$ 末端的位姿

Fig.10 The pose of capturing point $a$ with respect to arm- $a$ 's end-effector during capturing

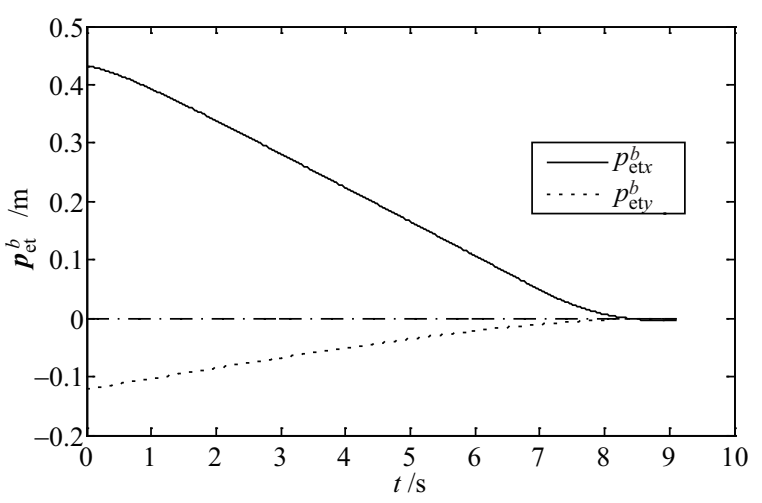

(a) The relative positon

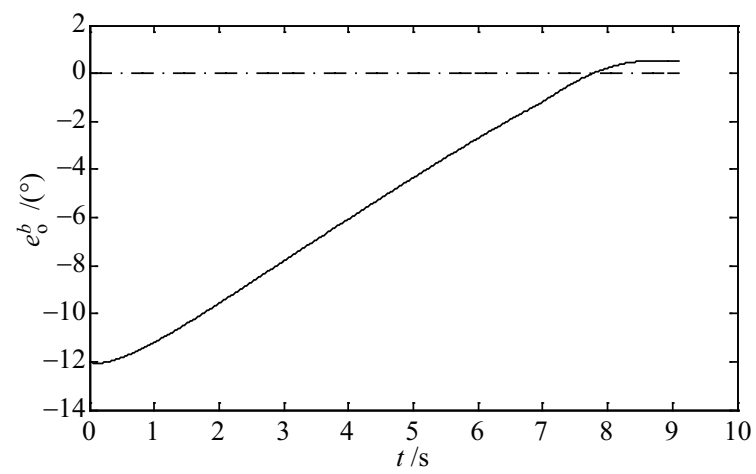

(b) The relative attitude

图 11 捕获过程中捕获点 $b$ 相对于 $a r m-b$ 末端的位置

Fig.11 The pose of capturing point $b$ with respect to arm- $b$ 's end-effector during capturing

此时满足抓捕条件，机械臂可闭合手爪抓捕 目标. 整个抓捕过程中, 基座姿态受控, 因此仅变 
化了 $-0.05^{\circ}$ (最后时刻); 基座质心位置不受控, 而是在耦合动力学作用下运动, 从 $[0,0.5 \mathrm{~m}]^{\mathrm{T}}$ 运 动到了 $[0.0093 \mathrm{~m}, 0.4820 \mathrm{~m}]^{\mathrm{T}}$, 即质心位置变化了 $[0.0093 \mathrm{~m},-0.0180 \mathrm{~m}]^{\mathrm{T}}$. 捕获时刻的状态如图 12 所示.

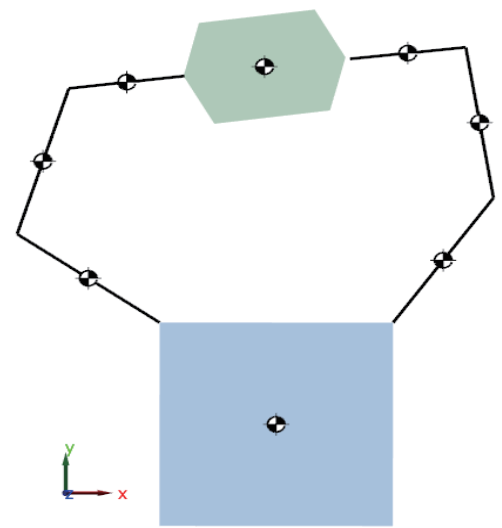

图 12 双臂协调捕获的最终状态

Fig.12 The final state of the dual-arm coordinated capturing

\section{6 结论 (Conclusion)}

本文主要针对抓捕过程三阶段的关键阶段— 接触碰撞前的跟踪接近（直到工具对目标上的被抓 捕结构进行包络的过程) 一一开展研究, 提出了相 应的基座自由漂浮和基座姿态受控两种模式下自主 轨迹规划方法. 该方法根据安装在机械臂末端的手 眼相机所提供的视觉测量结果, 自主规划、控制双 臂的运动，使其同时捕获目标航天器; 双臂协调捕 获能使捕获后形成的复合体系统迅速稳定，且抓捕 后的连接刚度高.

由于“接触碰撞”过程的动力学效应以及锁紧后 系统的状态依赖于具体的手爪及目标上被捕获部分 的具体结构和机构, 因此, 抓捕过程后两阶段一一接 触碰撞及捕获后系统稳定的动力学特性一一需要结 合实际情况具体分析, 未来将对这两阶段开展系统、 深入的研究.

\section{参考文献（References）}

[1] Boning P, Dubowsky S. Coordinated control of space robot teams for the on-orbit construction of large flexible space structures[J]. Advanced Robotics, 2010, 24(3): 303-323.

[2] Coleshill E, Oshinowo L, Rembala R, et al. Dextre: Improving maintenance operations on the international space station $[\mathrm{J}]$. Acta Astronautica, 2009, 64(9/10): 869-874.

[3] Diftler M A, Mehling J S, Abdallah M E, et al. Robonaut 2 - The first humanoid robot in space[C]//IEEE International Conference on Robotics and Automation. Piscataway, NJ, USA: IEEE, 2011: 2178-2183.

[4] Sabelli E, Akin D L, Carignan C R. Selecting impedance parameters for the ranger 8 -DoF dexterous space manipula-
tor[C]//AIAA Aerospace Conference and Exhibit. Reston, VA, USA: AIAA, 2007: 1-15.

[5] Debus T J, Dougherty S P. Overview and performance of the front-end robotics enabling near-term demonstration (FREND) robotic arm[C]//AIAA Infotech@Aerospace Conference. Reston, VA, USA: AIAA, 2009: AIAA 2009-1870,

[6] Papadopoulous E, Moosavian S A A. Dynamics and control of multi-arm space robots during chase and capture operations[C]//IEEE/RSJ/GI International Conference on Intelligent Robots and Systems. Piscataway, NJ, USA: IEEE, 1994: 15541561.

[7] Moosavian S A A, Evangelos S, Papadopoulos E. On the kinematics of multiple manipulator space free-flyers and their computation[J]. Journal of Robotic Systems, 1998, 15(4): 207-216.

[8] Rastegari R, Moosavian S A A. Multiple impedance control of space free-flying robots via virtual linkages[J]. Acta Astronautica, 2010, 66(5/6): 748-759.

[9] Hu Y R, Vukovich G. Dynamic control of free-floating coordinated space robots[J]. Journal of Robotic Systems, 1998, 15(4): 217-230.

[10] 田志祥, 吴洪涛. 闭环双臂空间机器人的动力学建模与仿真 [J]. 华南理工大学学报: 自然科学版, 2011, 39(8): 42-47,65.

Tian Z X, Wu H T. Dynamic modeling and simulation of closedloop dual-arm space robots[J]. Journal of South China University of Technology: Natural Science Edition, 2011, 39(8): 4247,65 .

[11] 陈志煌, 陈力. 闭链双臂空间机器人抓持载荷基于径向基 函数神经网络的补偿控制 [J]. 机械工程学报, 2011，47(7): 38-44.

Chen Z H, Chen L. Compensation control for grasped object of dual-arm space robot with closed-chain based on radial basis function neural network[J]. Chinese Journal of Mechanical Engineering, 2011, 47(7): 38-44.

[12] 王从庆, 张承龙. 自由浮动柔性双臂空间机器人系统的动 力学控制 [J]. 机械工程学报, 2007, 43(10): 196-200.

Wang C Q, Zhang C L. Dynamic control of a free-floating flexible dual-arm space robotic system[J]. Chinese Journal of Mechanical Engineering. 2007, 43(10): 196-200.

[13] Agrawal S K, Garimella R, Desmier G. Free-floating closedchain planar robots: Kinematics and path planning[J]. Nonlinear Dynamics, 1996, 9(1/2): 1-19.

[14] Chen L, Guo Y S. Hierarchical nonholonomic path planning of dual-arm space robot systems with joint limits[C]//The 6th World Congress on Intelligent Control and Automation. Piscataway, NJ, USA: IEEE, 2006: 8862-8865.

[15] 郭益深, 陈力. 闭环双臂空间机器人运动规划的双向逼近 方法 [J]. 力学与实践, 2007, 29(5): 7-11.

Guo Y S, Chen L. Bi-directional approach of motion planning of closed-loop space robot system with dual-arms[J]. Mechanics in Engineering, 2007, 29(5): 7-11.

[16] Wu H, Sun F C, Sun Z Q, et al. Optimal trajectory planning of a flexible dual-arm space robot with vibration reduction[J]. Journal of Intelligent and Robotic Systems, 2004, 40(2): 147-163.

[17] Nenchev D N, Yoshida K. Impact analysis and post-impact motion control issues of a free-floating space robot subject to a force impulse[J]. IEEE Transactions on Robotics and Automation, 1999, 15(3): 548-557. 
[18] 介党阳, 倪风雷, 谭益松, 等. 空间网状捕获接口的等效抓 捕控制 [J]. 机器人, 2011, 33(5): 534-538,627.

Jie D Y, Ni F L, Tan Y S, et al. Equivalent capture control of a space netting interface[J]. Robot, 2011, 33(5): 533-538,627.

[19] 张庆利, 倪风雷, 朱映远, 等. 三手指空间机器人末端执行 器的柔顺抓握策略 [J]. 机器人, 2011, 33(4): 427-433.

Zhang Q L, Ni F L, Zhu Y Y, et al. Compliant grasp strategy for three-fingered space robot end-effector[J]. Robot, 2011, 33(4): 427-433.

[20] Sagara S, Hideura M, Katoh R, et al. Adaptive RMRC for cooperative manipulation of a floating object by two free-based space robots[C]//IEEE/RSJ International Conference on Intelligent Robots and Systems. Piscataway, NJ, USA: IEEE, 1998: 1467-1472.

[21] Yamano M, Konno A, Uchiyama M. Experiments on capturing a floating object by two flexible manipulators[C]//IEEE International Conference on Robotics and Automation. Piscataway, NJ, USA: IEEE, 2000: 482-487.

[22] 郭琦, 洪炳镕, 王晶. 基于 Vega 的双臂空间机器人飞行及 捕捉目标的轨迹规划仿真 [J]. 机器人, 2005, 27(5): 385389,395 .

Guo Q, Hong B R, Wang J. Vega-based trajectory simulation for dual-arm space robot to fly and capture an object[J]. Robot, 2005, 27(5): 385-389,395.
[23] Takahashi R, Ise H, Konno A, et al. Hybrid simulation of a dualarm space robot colliding with a floating object[C]//LEEE International Conference on Robotics and Automation. Piscataway, NJ, USA: IEEE, 2008: 1201-1206.

[24] Cong P C, Sun Z W. Preimpact configuration analysis of dual-arm space manipulator capturing object[J]. Journal of Aerospace Engineering, 2010, 23(2): 117-123.

[25] Chiaverini S, Siciliano B, Egeland O. Review of the damped least-squares inverse kinematics with experiments on an industrial robot manipulator[J]. IEEE Transactions on Control Systems Technology, 1994, 2(2): 123-134.

[26] Xu W F, Liang B, Xu Y S. Practical approaches to handle the singularities of a wrist-partitioned space manipulator[J]. Acta Astronautica, 2011, 68(1/2): 269-300.

\section{作者简介:}

徐文福 (1979-), 男, 博士, 副教授. 研究领域: 空间机器 人, 视觉伺服控制.

刘厚德 (1984-), 男, 博士生. 研究领域: 空间机器人, 动 力学与控制.

李 成 (1972-), 男, 博士, 教授. 研究领域: 空间机器人, 微小卫星技术.

\section{附录 部分公式的详细表达式}

$$
\begin{aligned}
& k_{\mathrm{v} x}=-m_{1}^{a}\left(b_{0}^{a} s_{0}^{a}+a_{1}^{a} s_{01}^{a}\right)-m_{2}^{a}\left(b_{0}^{a} s_{0}^{a}+l_{1}^{a} s_{01}^{a}+a_{2}^{a} s_{012}^{a}\right)-m_{3}^{a}\left(b_{0}^{a} s_{0}^{a}+l_{1}^{a} s_{01}^{a}+l_{2}^{a} s_{012}^{a}+a_{3}^{a} s_{0123}^{a}\right)- \\
& m_{1}^{b}\left(b_{0}^{b} s_{0}^{b}+a_{1}^{b} s_{01}^{b}\right)-m_{2}^{b}\left(b_{0}^{b} s_{0}^{b}+l_{1}^{b} s_{01}^{b}+a_{2}^{b} s_{012}^{b}\right)-m_{3}^{b}\left(b_{0}^{b} s_{0}^{b}+l_{1}^{b} s_{01}^{b}+l_{2}^{b} s_{012}^{b}+a_{3}^{b} s_{0123}^{b}\right) \\
& k_{\mathrm{vy}}=m_{1}^{a}\left(b_{0}^{a} c_{0}^{a}+a_{1}^{a} c_{01}^{a}\right)+m_{2}^{a}\left(b_{0}^{a} c_{0}^{a}+l_{1}^{a} c_{01}^{a}+a_{2}^{a} c_{012}^{a}\right)+m_{3}^{a}\left(b_{0}^{a} c_{0}^{a}+l_{1}^{a} c_{01}^{a}+l_{2}^{a} c_{012}^{a}+a_{3}^{a} c_{0123}^{a}\right)+ \\
& m_{1}^{b}\left(b_{0}^{b} c_{0}^{b}+a_{1}^{b} c_{01}^{b}\right)+m_{2}^{b}\left(b_{0}^{b} c_{0}^{b}+l_{1}^{b} c_{01}^{b}+a_{2}^{b} c_{012}^{b}\right)+m_{3}^{b}\left(b_{0}^{b} c_{0}^{b}+l_{1}^{b} c_{01}^{b}+l_{2}^{b} c_{012}^{b}+a_{3}^{b} c_{0123}^{b}\right) \\
& k_{\mathrm{p} x 1}^{k}=-m_{1}^{k} a_{1}^{k} s_{01}^{k}-m_{2}^{k}\left(l_{1}^{k} s_{01}^{k}+a_{2}^{k} s_{012}^{k}\right)-m_{3}^{k}\left(l_{1}^{k} s_{01}^{k}+l_{2}^{k} s_{012}^{k}+a_{3}^{k} s_{0123}^{k}\right) \\
& k_{\mathrm{p} x 2}^{k}=-m_{2}^{k} a_{2}^{k} s_{012}^{k}-m_{3}^{k}\left(l_{2}^{k} s_{012}^{k}+a_{3}^{k} s_{0123}^{k}\right) \\
& k_{\mathrm{p} x 3}^{k}=-m_{3}^{k} a_{3}^{k} s_{0123}^{k} \\
& k_{\mathrm{py} 1}^{k}=m_{1}^{k} a_{1}^{k} c_{01}^{k}+m_{2}^{k}\left(l_{1}^{k} c_{01}^{k}+a_{2}^{k} c_{012}^{k}\right)+m_{3}^{k}\left(l_{1}^{k} c_{01}^{k}+l_{2}^{k} c_{012}^{k}+a_{3}^{k} c_{0123}^{k}\right) \\
& k_{\mathrm{py} 2}^{k}=m_{2}^{k} a_{2}^{k} c_{012}^{k}+m_{3}^{k}\left(l_{2}^{k} c_{012}^{k}+a_{3}^{k} c_{0123}^{k}\right) \\
& k_{\mathrm{p} y 3}^{k}=m_{3}^{k} a_{3}^{k} c_{0123}^{k} \\
& k_{\omega}=\left(I_{0}+I_{1}^{a}+I_{2}^{a}+I_{1}^{b}+I_{2}^{b}\right)+m_{1}^{a}\left[\left(b_{0}^{a} c_{0}^{a}+a_{1}^{a} c_{01}^{a}\right)^{2}+\left(b_{0}^{a} s_{0}^{a}+a_{1}^{a} s_{01}^{a}\right)^{2}\right]+ \\
& m_{2}^{a}\left[\left(b_{0}^{a} c_{0}^{a}+l_{1}^{a} c_{01}^{a}+a_{2}^{a} c_{012}^{a}\right)^{2}+\left(b_{0}^{a} s_{0}^{a}+l_{1}^{a} s_{01}^{a}+a_{2}^{a} s_{012}^{a}\right)^{2}\right]+ \\
& m_{3}^{a}\left[\left(b_{0}^{a} c_{0}^{a}+l_{1}^{a} c_{01}^{a}+l_{2}^{a} c_{012}^{a}+a_{3}^{a} c_{0123}^{a}\right)^{2}+\left(b_{0}^{a} s_{0}^{a}+l_{1}^{a} s_{01}^{a}+l_{2}^{a} s_{012}^{a}+a_{3}^{a} s_{0123}^{a}\right)^{2}\right]+ \\
& m_{1}^{b}\left(b_{0}^{b} c_{0}^{b}+a_{1}^{b} c_{01}^{b}\right)^{2}+\left(b_{0}^{b} s_{0}^{b}+a_{1}^{b} s_{01}^{b}\right)^{2}+m_{2}^{b}\left(b_{0}^{b} c_{0}^{b}+l_{1}^{b} c_{01}^{b}+a_{2}^{b} c_{012}^{b}\right)^{2}+\left(b_{0}^{b} s_{0}^{b}+l_{1}^{b} s_{01}^{b}+a_{2}^{b} s_{012}^{b}\right)^{2}+ \\
& m_{3}^{b}\left[\left(b_{0}^{b} c_{0}^{b}+l_{1}^{b} c_{01}^{b}+l_{2}^{b} c_{012}^{b}+a_{3}^{b} c_{0123}^{b}\right)^{2}+\left(b_{0}^{b} s_{0}^{b}+l_{1}^{b} s_{01}^{b}+l_{2}^{b} s_{012}^{b}+a_{3}^{b} s_{0123}^{b}\right)^{2}\right] \\
& k_{q 1}^{k}=\left(I_{1}^{k}+I_{2}^{k}+I_{3}^{k}\right)+m_{1}^{k}\left[a_{1}^{k} c_{01}^{k}\left(b_{0}^{k} c_{0}^{k}+a_{1}^{k} c_{01}^{k}\right)+a_{1}^{k} s_{01}^{k}\left(b_{0}^{k} s_{0}^{k}+a_{1}^{k} s_{01}^{k}\right)\right]+ \\
& m_{2}^{k}\left[\left(b_{0}^{k} c_{0}^{k}+l_{1}^{k} c_{01}^{k}+a_{2}^{k} c_{012}^{k}\right)\left(l_{1}^{k} c_{01}^{k}+a_{2}^{k} c_{012}^{k}\right)+\left(b_{0}^{k} s_{0}^{k}+l_{1}^{k} s_{01}^{k}+a_{2}^{k} s_{012}^{k}\right)\left(l_{1}^{k} s_{01}^{k}+a_{2}^{k} s_{012}^{k}\right)\right]+ \\
& m_{3}^{k}\left[\left(b_{0}^{k} c_{0}^{k}+l_{1}^{k} c_{01}^{k}+l_{2}^{k} c_{012}^{k}+a_{3}^{k} c_{0123}^{k}\right)\left(l_{1}^{k} c_{01}^{k}+l_{2}^{k} c_{012}^{k}+a_{3}^{k} c_{0123}^{k}\right)+\right. \\
& \left.\left(b_{0}^{k} s_{0}^{k}+l_{1}^{k} s_{01}^{k}+l_{2}^{k} s_{012}^{k}+a_{3}^{k} s_{0123}^{k}\right)\left(l_{1}^{k} s_{01}^{k}+l_{2}^{k} s_{012}^{k}+a_{3}^{k} s_{0123}^{k}\right)\right]
\end{aligned}
$$


图 7 是在以上参数和路径规划下 FROG-LEG 型 机器人单只手臂水平方向的扭矩变化情况. 从以上 的计算方法和仿真结果可以看出, 自然正交补方法 的运用使得机器人的动力学变得相对简洁, 物理意 义也更加明确.

\section{5 结论 (Conclusions)}

传统的 Euler-Lagrange 方程或 Newton-Euler 递 推法等求解并联机器人动力学较为复杂, 且容易出 错. 本文针对 FROG-LEG 型真空并联机器人, 提出 了该类机器人的等效串联模型, 并利用基于自然正 交补的方法对真空并联机器人进行了动力学建模, 通过计算和仿真证明了方法在降低求解难度和复杂 度方面的有效性.

\section{参考文献（References）}

[1] Quirk M, Serda J. 半导体制造技术 [M]. 韩郑生, 译. 北京： 电子工业出版社, 2009: 4-15.

Quirk M, Serda J. Semiconductor manufacturing technology[M]. Han Z S, trans. Beijing: Publishing House of Electronics Industry, 2009: 4-15.

[2] Cong M, Zhou Y M, Jiang Y. An automated wafer-handling system based on the integrated circuit equipments[C]//IEEE International Conference on Robotics and Biomimetics. Piscataway, NJ, USA: IEEE, 2005: 240-245.

[3] 黄裕仁. 晶圆输送机器人臂之机构分析及运动控制研究 [D]. 台湾: 台湾中原大学, 1999.

Huang Y R. Mechanism analysis and motion control of wafer transfer arm[D]. Taiwan: Chung Yuan Christian University, 1999.

[4] Uicker J. Dynamic behavior of spatial linkage[J]. ASME Mechanisms, 1969, 5(68): 1-15.

[5] Luh J Y S, Walker M W, Paul R P C. On-line computational scheme for mechanical manipulators[J]. Transactions of the
ASME, Journal of Dynamic Systems, Measurement and Control, 1980, 102(2): 69-76.

[6] Angeles J. 机器人机械系统原理理论、方法和算法 [M]. 宋 伟刚，译. 北京: 机械工业出版社, 2007: 168-178.

Angeles J. Fundamentals of robotic mechanical systems: Theory, methods, and algorithms[M]. Song W G, trans. Beijing: Machinery Industry Press, 2007: 168-178.

[7] 黄真, 赵永生, 赵铁石. 高等空间机构学 [M]. 北京: 高等 教育出版社, 2006: 224-228.

Huang Z, Zhao Y S, Zhao T S. Advanced spatial mechanism[M]. Beijing: Higher Education Press, 2006: 224-228.

[8] Parasuraman S, Liang P C J. Development of RPS parallel manipulators[C]//2nd International Conference on Computer and Network Technology. Piscataway, NJ, USA: IEEE, 2010: 600605.

[9] 黄昔光, 何广平, 谭晓兰, 等. 并联机器人机构研究现状分 析 [J]. 北方工业大学学报, 2009, 21(3): 25-30.

Huang X G, , He G P, Tian X L, et al. Parallel mechanism research status analysis[J]. Journal of North China University of Technology, 2009, 21(3): 25-30.

[10] Craig J. Introduction to robotics: Mechanics and control[M]. Englewood Cliffs, NJ, USA: Prentice Hall, 2004: 48-70.

[11] 蔡自兴. 机器人学 [M]. 北京: 清华大学出版社, 2000: 37-39. Cai Z X. Robotics[M]. Beijing: Tsinghua University Press, 2000: $37-39$

[12] Denavit J, Hartenberg R S. A kinematic notation for lower-pair mechanisms based on matrices[J]. Journal of Applied Mechanics, 1955, 22(1): 215-221.

[13] 日本机器人学会. 新版机器人技术手册 [M]. 宗光华, 程君 实，译. 2 版. 北京: 科学出版社, 2008: 508-601.

Japan Robot Association. The new handbook of robot technology[M]. Zong G H, Cheng J S, trans. 2nd ed. Beijing: Science Press, 2008: 508-601.

\section{作者简介:}

黄玉钏 (1986-), 男, 博士生. 研究领域: 机器人学, 机电 控制.

$$
\begin{aligned}
k_{q 2}^{k}= & \left(I_{2}^{k}+I_{3}^{k}\right)+m_{2}^{k}\left[\left(b_{0}^{k} c_{0}^{k}+l_{1}^{k} c_{01}^{k}+a_{2}^{k} c_{012}^{k}\right) a_{2}^{k} c_{012}^{k}+\left(b_{0}^{k} s_{0}^{k}+l_{1}^{k} s_{01}^{k}+a_{2}^{k} s_{012}^{k}\right) a_{2}^{k} s_{012}^{k}\right]+ \\
& m_{3}^{k}\left[\left(b_{0}^{k} c_{0}^{k}+l_{1}^{k} c_{01}^{k}+l_{2}^{k} c_{012}^{k}+a_{3}^{k} c_{0123}^{k}\right)\left(l_{2}^{k} c_{012}^{k}+a_{3}^{k} c_{0123}^{k}\right)+\right. \\
& \left.\quad\left(b_{0}^{k} s_{0}^{k}+l_{1}^{k} s_{01}^{k}+l_{2}^{k} s_{012}^{k}+a_{3}^{k} s_{0123}^{k}\right)\left(l_{2}^{k} s_{012}^{k}+a_{3}^{k} s_{0123}^{k}\right)\right] \\
k_{q 3}^{k}= & I_{3}^{k}+m_{3}^{k}\left[\left(b_{0}^{k} c_{0}^{k}+l_{1}^{k} c_{01}^{k}+l_{2}^{k} c_{012}^{k}+a_{3}^{k} c_{0123}^{k}\right) a_{3}^{k} c_{0123}^{k}+\left(b_{0}^{k} s_{0}^{k}+l_{1}^{k} s_{01}^{k}+l_{2}^{k} s_{012}^{k}+a_{3}^{k} s_{0123}^{k}\right) a_{3}^{k} s_{0123}^{k}\right]
\end{aligned}
$$

